Existence and approximation of solutions of some first order iterative differential equations

\author{
Vasile Berinde
}




\title{
EXISTENCE AND APPROXIMATION OF SOLUTIONS OF SOME FIRST ORDER ITERATIVE DIFFERENTIAL EQUATIONS
}

\author{
VASILE BERINDE
}

Received 28 December, 2009

\begin{abstract}
Existence theorems for some iterative differential equations as well as convergence theorems for a fixed point iterative method designed to approximate these solutions, are proved under weaker conditions than those due to A. Buică ("Existence and continuous dependence of solutions of some functional-differential equations," Seminar on Fixed Point Theory 3, pp. 1-14, 1995). To this end, we basically employ the technique of non-expansive mappings.
\end{abstract}

2000 Mathematics Subject Classification: 34A12, 39B12, 47H09

Keywords: iterative differential equation, solution, non-expansive operator, fixed point, convergence theorem

\section{INTRODUCTION}

For most of the differential and integral equations with deviating arguments that appear in recent literature, the deviation of the argument usually involves only the time itself, see for example the very recent papers [1, 2, 6, 18, 19, 22, 23]. However, another case, in which the deviating arguments depend on both the state variable $x$ and the time $t$, is of importance in theory and practice. Several papers have appeared recently that are devoted to such kind of differential equations, see for example [4,8$12,16,17,29,31-33]$ and references therein.

One of the first papers studying this class of functional equations is the one by Eder [8] who considered the functional differential equation

$$
x^{\prime}(t)=x(x(t)), \quad t \in A \subset \mathbb{R},
$$

while Fečkan [12] studied a functional differential equation of the more general form

$$
x^{\prime}(t)=f(x(x(t)))
$$

with $f \in C^{1}(\mathbb{R})$. For other developments on this topic, see the very recent papers $[4,8-11,16,17,29,33]$ and references therein. The term to designate this class of differential equations is that of iterative differential equations. As mentioned in [33] and the papers cited there, iterative differential equations arise in relation to infection 
models and are also important in the study of the motion of charged particles with retarded interaction.

In this paper, we are interested in the study of the existence of solutions of iterative differential equations of the general form

$$
x^{\prime}(t)=f(t, x(x(t))),
$$

and, in addition to other related papers, we illustrate how one can approximate the non-unique solution of such kind of iterative differential equations by means of iterative techniques.

The main idea is to use the powerful and more reliable technique of non-expansive operators and to adapt and use several convergence theorems from the theory of iterative approximation of fixed points of non-expansive mappings (see the very recent monographs $[3,5])$.

As most of the papers in literature devoted to functional differential equations with deviating argument are based on fixed point techniques and are essentially tributary to the contraction mapping principle or method of successive approximations, our approach here appears to be new not only for the study of existence of solutions but especially with regard to the approximation of solutions of these equations.

The paper is organized as follows: in Section 2 we present some basic results from the fixed point theory of non-expansive operators; in Section 3 we present the main results of the paper regarding the existence and approximation of solutions of some iterative differential equations. The paper ends with Section 4 that gives some illustrative examples. Note that our results are established under weaker assumptions than the ones obtained in [4].

\section{FIXED POINT THEORY OF NON-EXPANSIVE MAPPINGS}

Let $(X, d)$ be a metric space. A mapping $T: X \rightarrow X$ is said to be an $\alpha$-contraction if there exists $\alpha \in[0,1)$ such that

$$
d(T x, T y) \leq \alpha d(x, y), \quad \forall x, y \in X .
$$

A point $x \in X$ is called a fixed point of $T$ if $T x=x$. It is well known [26] that, under the strict contraction condition (2.1) in a complete metric space $X$, there exists a unique fixed point of $T$ and, moreover, the Picard iteration determined by an $x_{0} \in X$ and the relation

$$
x_{n+1}=T x_{n}, \quad n=0,1,2, \ldots,
$$

converges to that fixed point. In the case where $\alpha=1$ in (2.1), the mapping $T$ is said to be non-expansive.

As the technique of non-expansive mappings applied to functional differential equations appears to be less frequently used in literature, in the present section we present some basic concepts and results of the fixed point theory of non-expansive operators. 
Let $K$ be a non-empty subset of a real normed linear space $E$ and let $T: K \rightarrow K$ be a mapping. In this setting, $T$ is non-expansive if

$$
\|T x-T y\| \leq\|x-y\|, \quad \forall x, y \in K .
$$

Although the non-expansive mappings are generalizations of $\alpha$-contractions, they of contractive mappings. More precisely, if $K$ is a non-empty closed subset of a Banach space $E$ and $T: K \rightarrow K$ is a non-expansive mapping which is not an $\alpha$-contraction, then, as is shown by the following example, $T$ may not have fixed points.

Example 1 ([13, Example 3.3, pp. 30]). In the space $c_{0}(\mathbb{N})$, the isometry $T$ defined by the relation

$$
T\left(x_{1}, x_{2}, \ldots\right)=\left(1, x_{1}, x_{2}, \ldots\right)
$$

maps the unit ball into its boundary but has no fixed points.

Moreover, as is shown by the next example, even in the cases where $T$ has a fixed point, the Picard iteration associated to $T$ (i. e., the sequence $\left\{x_{n}\right\}$ defined by (2.2) for an $x_{0} \in K$ ), may fail to converge to the fixed point.

Example 2. Let us consider the unit interval $[0,1]$ with the usual norm. The function $T:[0,1] \rightarrow[0,1]$ given by the formula $T x=1-x$ for all $x \in[0,1]$ has a unique fixed point, $x^{*}=\frac{1}{2}$ but, except for the trivial case $x_{0}=\frac{1}{2}$, the Picard iteration starting from $x_{0}$ yields an oscillatory sequence.

For this many other reasons, some richer geometrical properties of the ambient space $E$ are needed in order to ensure the existence of a fixed point or/and the convergence of an iterative method (generally a more complex iterative method than the Picard iteration) to a fixed point of $T$. In the present paper, we mainly consider Banach spaces which are uniformly convex or strictly convex (for more general settings, see [5]). For the sake of completeness, let us recall some concepts and results.

One of the most important fixed point theorems for non-expansive mappings, due to Browder, Göhde, and Kirk (see, e. g., [3]), is stated as follows.

Theorem 2.1. If $K$ is a non-empty closed convex and bounded subset of a uniformly convex Banach space $E$ then any non-expansive mapping $T: K \rightarrow K$ has a fixed point.

Remark 1. Theorem 2.1 provides no information on the approximation of a fixed point of $T$. From Example 2, we see that the Picard iteration does not resolve this situation, in general. Due to this fact, several other fixed point iteration procedures have been considered (see $[3,5])$. The most usual ones will be defined in the sequel in view of their use.

Let $K$ be a convex subset of a normed linear space $E$ and let $T: K \rightarrow K$ be a selfmapping. Given an $x_{0} \in K$ and a real number $\lambda \in[0,1]$, the sequence $\left\{x_{n}\right\}$ defined by the formula

$$
x_{n+1}=(1-\lambda) x_{n}+\lambda T x_{n}, \quad n=0,1,2, \ldots,
$$


is usually called the Krasnoselskij iteration, or Krasnoselskij-Mann iteration. Clearly, (2.4) reduces to the Picard iteration (2.2) for $\lambda=1$.

For an $x_{0} \in K$, the sequence $\left\{x_{n}\right\}$ defined by the formula

$$
x_{n+1}=\left(1-\lambda_{n}\right) x_{n}+\lambda_{n} T x_{n}, \quad n=0,1,2, \ldots,
$$

where $\left\{\lambda_{n}\right\} \subset[0,1]$ is a sequence of real numbers satisfying some appropriate conditions, is called a Mann iteration.

It was shown by Krasnoselskij [15] in the case where $\lambda=1 / 2$, and later by Schaefer [30] for an arbitrary $\lambda \in(0,1)$, that if $E$ is a uniformly convex Banach space and $K$ is a convex and compact subset of $E$ (and therefore, by Theorem 2.1, containing fixed points of $T$ ), then the Krasnoselskij iteration converges to a fixed point of $T$.

Moreover, Edelstein [7] proved that strict convexity of $E$ suffices for the same conclusion. The question of whether the strict convexity assumption can be removed was answered in the affirmative by Ishikawa [14] by the following result.

Theorem 2.2. Let $K$ be a subset of a Banach space $E$ and let $T: K \rightarrow K$ be a nonexpansive mapping. For an arbitrary $x_{0} \in K$, consider the Mann iteration process $\left\{x_{n}\right\}$ given by (2.5) under the following assumptions:

(a) $x_{n} \in K$ for all positive integers $n$;

(b) $0 \leq \lambda_{n} \leq b<1$ for all positive integers $n$;

(c) $\sum_{n=0}^{\infty} \lambda_{n}=\infty$.

If $\left\{x_{n}\right\}$ is bounded, then $x_{n}-T x_{n} \rightarrow 0$ as $n \rightarrow \infty$.

The following corollaries of Theorem 2.2 will be particularly important for the application part of our paper.

Corollary 2.1. Let $K$ be a convex and compact subset of a Banach space $E$ and let $T: K \rightarrow K$ be a non-expansive mapping. If the Mann iteration process $\left\{x_{n}\right\}$ given by (2.5) satisfies assumptions (a)-(c) of Theorem 2.2, then $\left\{x_{n}\right\}$ converges strongly to a fixed point of $T$.

Proof. See Theorem 6.17 in Chidume [5].

Corollary 2.2. Let $E$ be a real normed space, $K$ a closed bounded convex subset of $E$ and let $T: K \rightarrow K$ be a non-expansive mapping. If $I-T$ maps closed bounded subsets of $E$ into closed subsets of $E$ and $\left\{x_{n}\right\}$ is the Mann iteration defined by (2.5) with $\left\{\lambda_{n}\right\}$ satisfying assumptions (a)-(c) of Theorem 2.2, then $\left\{x_{n}\right\}$ converges strongly to a fixed point of $T$ in $K$.

Proof. See Corollary 6.19 in Chidume [5]. 


\section{EXISTENCE THEOREMS AND APPROXIMATION OF SOLUTIONS OF SOME ITERATIVE DIFFERENTIAL EQUATIONS}

Consider the following initial value problem

$$
\left\{\begin{array}{l}
y^{\prime}(x)=f(x, y(y(x))), \quad x \in[a, b], \\
y\left(x_{0}\right)=y_{0},
\end{array}\right.
$$

where $x_{0}, y_{0} \in[a, b]$ and $f \in C([a, b] \times[a, b])$ are given. Let us put $C_{x}=\max \{x-$ $a, b-x\}, x \in[a, b]$, and

$$
\mathscr{C}_{L}=\left\{y \in C([a, b],[a, b]):\left|y\left(t_{1}\right)-y\left(t_{2}\right)\right| \leq L\left|t_{1}-t_{2}\right|, \forall t_{1}, t_{2} \in[a, b]\right\},
$$

where $L>0$ is given. For problem (3.1), Buică [4] established existence theorems [4, Theorems 1 and 4] as well as existence and uniqueness theorems [4, Theorems 2 and 5]. We formulate the first two of them here for the sake of completeness.

Theorem 3.1 ([4]). Assume that the following conditions are satisfied for the initial value problem (3.1):

(1) $f \in C([a, b] \times[a, b])$;

(2) $\left.\exists L_{1}>0:|f(s, u)-f(s, v)| \leq L_{1} \mid u-v\right) \mid$ for all $s, u, v \in[a, b]$;

(3) If $L$ is the Lipschitz constant involved in (3.2), then

$$
M=\max \{|f(s, u)|:(s, u) \in[a, b] \times[a, b]\} \leq L ;
$$

(4) One of the following conditions holds:
(a) $M C_{x_{0}} \leq C_{y_{0}}$;
(b) $x_{0}=a, M(b-a) \leq b-y_{0}, f(s, u) \geq 0$ for all $s, u \in[a, b]$;
(c) $x_{0}=b, M(b-a) \leq y_{0}-a, f(s, u) \geq 0$ for all $s, u \in[a, b]$.

Then there exists at least one solution $y^{*} \in \mathscr{C}_{L}$ of problem (3.1).

Basically, Theorem 3.1 shows that, for any given $L>0$, if (1)-(4) are satisfied, then the initial value problem (3.1) has a (possibly, non-unique) solution in $\mathscr{C}_{L}$.

Theorem 3.2 ([4]). Assume that all conditions of Theorem 3.1 are satisfied and, in addition, we also have

$$
L_{1} C_{x_{0}}(L+1)<1 .
$$

Then there exists a unique solution $y^{*}$ of problem (3.1) in $\mathscr{C}_{L}$.

Under the assumptions of Theorem 3.2, it is known that the unique solution $y^{*}$ of the initial value problem (3.1) can be approximated by means of the Picard iteration $\left\{y_{n}\right\}$ defined by $y_{1} \in \mathscr{C}_{L}$ arbitrary and

$$
y_{n+1}(t)=y_{0}+\int_{x_{0}}^{t} f\left(s, y_{n}\left(y_{n}(s)\right)\right) d s, \quad \forall t \in[a, b], n \geq 1 .
$$


In view of the considerations presented in Section 2, it is clear that if condition (3.3) is weakened to

$$
L_{1} C_{x_{0}}(L+1) \leq 1
$$

then, firstly, the assertion on the existence of a unique solution of problem (3.1) is not true any more and, secondly, the Picard iteration (3.4) does not generally converge to the solution.

It is therefore the aim of this paper to show that if (3.3) is replaced by (3.5), then we are still able to approximate a (non-unique) solution of the initial value problem (3.1) by means of a Krasnoselski-Mann iteration procedure. The next theorem states the main result of this paper.

Theorem 3.3. Assume that all conditions of Theorem 3.1 are satisfied and, in addition, we have

$$
L_{1} C_{x_{0}}(L+1) \leq 1 .
$$

Then the initial value problem (3.1) has at least one solution $y^{*}$ in $\mathscr{C}_{L}$ which can be approximated by the Krasnoselskij iteration

$$
y_{n+1}(t)=(1-\mu) y_{n}(t)+\mu y_{0}+\mu \int_{x_{0}}^{t} f\left(s, y_{n}\left(y_{n}(s)\right)\right) d s, \quad t \in[a, b], n \geq 1,
$$

where $\mu \in(0,1)$ and $y_{1} \in \mathscr{C}_{L}$ is arbitrary.

Proof. If $L_{1} C_{x_{0}}(L+1)<1$, then the conclusion follows similarly to [4]. Therefore, we limit ourselves to the case where $L_{1} C_{x_{0}}(L+1)=1$.

It follows from [4, Lemma 1] that $\mathscr{C}_{L}$ is a non-empty convex and compact subset of the Banach space $(C([a, b]),\|\cdot\|)$, where $\|\cdot\|$ is the usual supremum norm. Consider the integral operator $T: \mathscr{C}_{L} \rightarrow C([a, b])$,

$$
T y(t)=y_{0}+\int_{x_{0}}^{t} f(s, y(y(s))) d s, \quad t \in[a, b], y \in \mathscr{C}_{L} .
$$

It is clear that $y \in \mathscr{C}_{L}$ is a solution of the initial value problem (3.1) if and only if $y$ is a fixed point of $T$, i. e.,

$$
y=T y .
$$

We first prove that $\mathscr{C}_{L}$ is an invariant set with respect to $T$, i. e., we have $T\left(\mathscr{C}_{L}\right) \subset \mathscr{C}_{L}$.

If condition (4a) holds, then for any $y \in \mathscr{C}_{L}$ and $t \in[a, b]$ we have

$$
|(T y)(t)| \leq\left|y_{0}\right|+\left|\int_{x_{0}}^{t} f(s, y(y(s))) d s\right| \leq\left|y_{0}\right|+M\left|t-x_{0}\right| \leq b
$$

and

$$
\begin{aligned}
|(T y)(t)| & \geq\left|y_{0}\right|-\left|\int_{x_{0}}^{t} f(s, y(y(s))) d s\right| \geq\left|y_{0}\right|-M\left|t-x_{0}\right| \\
& \geq\left|y_{0}\right|-M C_{x_{0}} \geq y_{0}-C_{y_{0}} \geq a
\end{aligned}
$$


which shows that, for any $y \in \mathscr{C}_{L}$, one has $(T y)(t) \in[a, b], t \in[a, b]$. Now, for any $t_{1}, t_{2} \in[a, b]$, we have

$$
\left|(T y)\left(t_{1}\right)-(T y)\left(t_{2}\right)\right|=\left|\int_{t_{1}}^{t_{2}} f(s, y(y(s))) d s\right| \leq M\left|t_{1}-t_{2}\right| \leq L\left|t_{1}-t_{2}\right| .
$$

Thus, $T y \in \mathscr{C}_{L}$ for all $y \in \mathscr{C}_{L}$. In a similar way we treat the cases (4b) and (4c). Therefore, $T: \mathscr{C}_{L} \rightarrow \mathscr{C}_{L}$ (i. e., $T$ is a self-mapping of $\mathscr{C}_{L}$ ).

Let $y, z \in \mathscr{C}_{L}$ and $t \in[a, b]$. Then

$$
\begin{aligned}
\mid(T y)(t)- & (T z)(t)|\leq| \int_{x_{0}}^{t}|f(s, y(y(s)))-f(s, z(z(s)))| d s \mid \\
& \leq\left|\int_{x_{0}}^{t} L_{1}\right| y(y(s))-z(z(s))|d s| \\
& \leq L_{1}\left|\int_{x_{0}}^{t}(|y(y(s))-y(z(s))|+|y(z(s))-z(z(s))|) d s\right| \\
& \leq L_{1}\left|\int_{x_{0}}^{t}\left(L|y(s)-z(s)|+\max _{\xi \in[a, b]}|y(\xi)-z(\xi)|\right) d s\right| \\
& =L_{1}\left|\int_{x_{0}}^{t}(L|y(s)-z(s)|+\|y-z\|) d s\right| \\
& \leq L_{1}\left|\int_{x_{0}}^{t}(L+1)\|y-z\|\right| d s \\
& =L_{1}(L+1)\|y-z\|\left|\| t-x_{0}\right| \\
& \leq L_{1} C_{x_{0}}(L+1)\|y-z\| .
\end{aligned}
$$

Now, by taking the maximum in (3.8), we get

$$
\|T y-T z\| \leq L_{1} C_{x_{0}}(L+1)\|y-z\|
$$

which, in view of condition (3.5), proves that $T$ is non-expansive and, hence, continuous.

It now remains to apply the Schauder fixed point theorem to obtain the first part of the conclusion, and Corollary 2.1 or 2.2 to get the second one.

Remark 2. In practise, one can consider $\mu=\frac{1}{2}$ in (3.6).

Now we state and prove the results corresponding to Theorems 4 and 5 of [4]. To this end, for a $\lambda \in(0,1]$ fixed, we put

$$
\mathscr{C}_{L, \lambda}=\left\{y \in \mathscr{C}_{L}: y(x) \leq \lambda x, \forall x \in[a, b]\right\} .
$$

Theorem 3.4. Assume that the following conditions are satisfied:

(i) $y_{0} \leq \lambda x_{0}$;

(ii) $\exists L_{1}>0:|f(s, u)-f(s, v)| \leq L_{1}|u-v|$ for all $s, u, v \in[a, b]$; 
(iii) $M \leq \min \{\lambda, L\}$;

(iv) One of the following conditions holds:

(a) $M C_{x_{0}} \leq C_{y_{0}}$;

(b) $x_{0}=a, M(b-a) \leq b-y_{0}$, and $f(s, u) \geq 0$ for all $s, u \in[a, b]$;

(c) $x_{0}=b, M(b-a) \leq y_{0}-a$, and $f(s, u) \geq 0$ for all $s, u \in[a, b]$;

(v) $M\left(x_{0}-a\right) \geq y_{0}-\lambda a$;

(vi) There exists a $\bar{\tau}>0$ such that $\bar{\tau}>-\frac{\ln (1-\lambda)}{\lambda\left(b-x_{0}\right)}$ (if $x_{0} \neq b$ and $\left.\lambda \neq 1\right)$ and

$$
\frac{L_{1}}{\bar{\tau}}\left(L+\frac{1}{\lambda}\right) \max \left\{e^{\bar{\tau}\left(x_{0}-a\right)}-1,1-e^{\bar{\tau}\left(x_{0}-b\right)}\right\} \leq 1 .
$$

Then:

(1) The initial value problem (3.1) has at least one solution in $\mathscr{C}_{L, \lambda}$;

(2) For any $y_{1} \in \mathscr{C}_{L, \lambda}$ the Krasnoselskij iteration

$$
y_{n+1}(t)=(1-\mu) y_{n}(t)+\mu y_{0}+\mu \int_{x_{0}}^{t} f\left(s, y_{n}\left(y_{n}(s)\right)\right) d s, \quad t \in[a, b], n \geq 1 \text {, }
$$

where $\mu \in(0,1)$, converges to a solution of problem (3.1) as $n \rightarrow \infty$.

Proof. In view of [4, Lemma 1], the set $\mathscr{C}_{L, \lambda}$ is a convex and compact subset of the Banach space $C[a, b]$ endowed with Bielecki's norm given by the formula

$$
\|y\|_{B}=\max _{x \in[a, b]}|y(x)| e^{-\tau\left(x-x_{0}\right)}
$$

for all $y \in C[a, b]$, where $x_{0} \in[a, b]$ and $\tau>0$ are fixed. Let $T$ be defined as in the proof of Theorem 3.3. By assumptions (ii), (iii), and (iv), it follows that

$$
T\left(\mathscr{C}_{L, \lambda}\right) \subset \mathscr{C}_{L} .
$$

Let us prove that $\mathscr{C}_{L, \lambda}$ is an invariant set with respect to the operator $T$. Indeed, if $y \in \mathscr{C}_{L, \lambda}$ and $x \in[a, b]$, we have

$$
\begin{aligned}
(T y)(x) & \leq y_{0}+M\left(x-x_{0}\right) \\
& =y_{0}+M(x-a)-M\left(x_{0}-a\right) \\
& \leq y_{0}+\lambda(x-a)-\left(y_{0}-\lambda a\right)=\lambda x,
\end{aligned}
$$

that is, $T y \in \mathscr{C}_{L, \lambda}$, where we have used (iii) and (v).

For $y, z \in \mathscr{C}_{L, \lambda}$ and $x \in[a, b]$, we have

$$
\begin{aligned}
\mid(T y)(x)- & (T z)(x)\left|\leq L_{1}\right| \int_{x_{0}}^{x}(L|y(s)-z(s)|+|y(z(s))-z(z(s))|) d s \mid \\
& \leq L_{1}\left(\left|\int_{x_{0}}^{x} L e^{\tau\left(s-x_{0}\right)} d s\right|+\left|\int_{x_{0}}^{x} e^{\tau\left(\lambda s-x_{0}\right)} d s\right|\right)\|y-z\|_{B} \\
& =L_{1}\left(\frac{L}{\tau}\left|e^{\tau\left(x-x_{0}\right)}-1\right|+\frac{1}{\tau \lambda}\left|e^{\tau\left(\lambda x-x_{0}\right)}-e^{\tau\left(\lambda x_{0}-x_{0}\right)}\right|\right)\|y-z\|_{B} .
\end{aligned}
$$


This shows that

$$
\begin{aligned}
\mid(T y)(x) & -(T z)(x) \mid e^{-\tau\left(x-x_{0}\right)} \\
& \leq L_{1}\left(\frac{L}{\tau}\left|1-e^{-\tau\left(x-x_{0}\right)}\right|+\frac{1}{\tau \lambda}\left|e^{\tau(\lambda x-x)}-e^{\tau\left(\lambda x_{0}-x\right)}\right|\right)\|y-z\|_{B} \\
& =\frac{L_{1}}{\tau}\left(L\left|1-e^{-\tau\left(x-x_{0}\right)}\right|+\frac{1}{\lambda}\left|e^{\tau(\lambda-1) x}-e^{\tau\left(\lambda x_{0}-x\right)}\right|\right)\|y-z\|_{B} \\
& =L_{T}(x)\|y-z\|_{B},
\end{aligned}
$$

where $L_{T}:[a, b] \rightarrow \mathbb{R}$ is a continuous function. Then there exists a constant $\tilde{L}_{T}>0$ such that

$$
\max _{x \in[a, b]} L_{T}(x) \leq \tilde{L}_{T}
$$

Thus, by (3.9) we get

$$
\|T y-T z\|_{B} \leq \widetilde{L}_{T}\|y-z\|_{B}
$$

which shows that $T$ is Lipschitzian, hence continuous. By Schauder's fixed point theorem it follows that $T$ has at least one fixed point $y^{*} \in \mathscr{C}_{L}$, which is actually a solution of the initial value problem (3.1).

To prove the second part of the theorem, we evaluate the maximum of $L_{T}(x)$. It is easy to prove that $g(x)=1-e^{-\tau\left(x-x_{0}\right)}$ is strictly increasing on $[a, b]$ and $g\left(x_{0}\right)=0$, so

$$
\max _{x \in[a, b]}|g(x)|=\max \left\{e^{\tau\left(x_{0}-a\right)}-1,1-e^{\tau\left(x_{0}-b\right)}\right\} .
$$

Similarly, if we set $h(x)=e^{\tau(\lambda-1) x}-e^{\tau\left(\lambda x_{0}-x\right)}$, then

$$
h^{\prime}(x)=\tau e^{\tau(\lambda-1) x}\left(\lambda-1+e^{-\tau \lambda\left(x-x_{0}\right)}\right) .
$$

It is clear that the function $h_{1}(x)=\lambda-1+e^{-\tau \lambda\left(x-x_{0}\right)}$ is strictly decreasing on $[a, b]$ and, hence, $h_{1}(x) \geq h_{1}(b)=\lambda-1+e^{-\tau \lambda\left(b-x_{0}\right)}$.

If $x_{0}=b$, then $h_{1}(b)=\lambda-1 \leq 0$, which shows that $h$ is decreasing on $[a, b]$. If $\lambda=1$, then $h_{1}(b)>0$ and so $h$ is strictly increasing on $[a, b]$. Finally, if $\lambda \neq 1$ and $x_{0} \neq b$, then, by assumption (vi), we can choose $\bar{\tau}>0$ such that

$$
\bar{\tau}>-\frac{\ln (1-\lambda)}{\lambda\left(b-x_{0}\right)}
$$

which implies that $h_{1}(b)>0$ and hence $h$ is strictly increasing on $[a, b]$. We put $\tau=\bar{\tau}$. Then, in each of the three cases,

$$
\max _{x \in[a, b]}|h(x)|=\max \left\{\left|e^{\tau(\lambda-1) a}-e^{\tau\left(\lambda x_{0}-a\right)}\right|,\left|e^{\tau(\lambda-1) b}-e^{\tau\left(\lambda x_{0}-b\right)}\right|\right\} .
$$

Using the fact that $\lambda \leq 1$, we have

$$
\left|e^{\tau(\lambda-1) a}-e^{\tau\left(\lambda x_{0}-a\right)}\right|=e^{\tau(\lambda-1) a}\left|1-e^{\tau \lambda\left(x_{0}-a\right)}\right|
$$




$$
\begin{aligned}
& =e^{\tau(\lambda-1) a}\left(e^{\tau \lambda\left(x_{0}-a\right)}-1\right) \\
& \leq e^{\tau \lambda\left(x_{0}-a\right)}-1 .
\end{aligned}
$$

Similarly,

$$
\begin{aligned}
\left|e^{\tau(\lambda-1) b}-e^{\tau\left(\lambda x_{0}-b\right)}\right| & =e^{\tau(\lambda-1) b}\left|1-e^{\tau \lambda\left(x_{0}-b\right)}\right| \\
& =e^{\tau(\lambda-1) b}\left(1-e^{\tau \lambda\left(x_{0}-b\right)}\right) \\
& \leq 1-e^{\tau \lambda\left(x_{0}-b\right)} .
\end{aligned}
$$

As a consequence of the results above, we have

$$
L_{T}(x) \leq \max \left\{e^{\tau\left(x_{0}-a\right)}-1,1-e^{\tau\left(x_{0}-b\right)}\right\} \frac{L_{1}}{\tau}\left(L+\frac{1}{\lambda}\right)
$$

for all $x \in[a, b]$, which, by (3.9), shows that $T$ is non-expansive. Now one can use Corollaries 2.1 and 2.2 to get the second part of the theorem.

\section{CONCLUDing REMARKS AND EXAMPLES}

Remark 3. Note that if one can find a $\tau>0$ such that

$$
\max \left\{e^{\tau\left(x_{0}-a\right)}-1,1-e^{\tau\left(x_{0}-b\right)}\right\} \frac{L_{1}}{\tau}\left(L+\frac{1}{\lambda}\right)<1,
$$

then, instead of considering the non-expansive mapping principle in Theorem 3.4, we can use the contraction mapping principle similarly to [4, Theorem 5].

Note also that condition (vi) of our Theorem 3.4 is slightly simpler and weaker than the corresponding one appearing in [4]:

$$
\max \left\{(\lambda-1) b,(\lambda-1) a, \frac{x_{0}-a}{\ln 2}, \frac{\lambda\left(x_{0}-a\right)}{\ln 2}\right\} L_{1}\left(L+\frac{e}{\lambda}\right)<1 .
$$

We conclude the paper by presenting two examples which illustrate the generality and efficiency of our results.

Example 3. Consider the following initial value problem associated to an iterative differential equation similar to the ones studied in [8,31],

$$
\left\{\begin{array}{l}
y^{\prime}(x)=-\frac{1}{2}+y(y(x)), \quad x \in[0,1], \\
y\left(\frac{1}{2}\right)=\frac{1}{2}
\end{array}\right.
$$

where $y \in C^{1}([0,1],[0,1])$.

We are interested in the solutions $y \in C^{1}([0,1],[0,1])$ belonging to the set

$$
\mathscr{C}_{1}=\left\{y \in C([0,1],[0,1]):\left|y\left(t_{1}\right)-y\left(t_{2}\right)\right| \leq\left|t_{1}-t_{2}\right|, \forall t_{1}, t_{2} \in[0,1]\right\},
$$

which, in view of our notation, means that $L=1$. We also have $a=0, b=1$, and $x_{0}=\frac{1}{2}$, hence $C_{x_{0}}=\max \left\{x_{0}-a, b-x_{0}\right\}=\frac{1}{2}$. 
The function $f(x, u)=-\frac{1}{2}+u$ is Lipschitzian with the Lipschitz constant $L_{1}=1$ (i. e., $f$ is non-expansive). This shows that

$$
L_{1} C_{x_{0}}(L+1)=1
$$

so condition (3.5) is satisfied but condition (3.3) is not. Therefore, by Theorem 3.3 (but not by Theorem 3.2!) we obtain information on the existence and approximation of the solutions of the initial value problem (4.1).

Note also that the function $y(x)=\frac{1}{2}, x \in[0,1]$, is a solution of the initial value problem (4.1).

Example 4. For the iterative differential equation

$$
y^{\prime}(x)=\frac{1}{10} y(y(t)), \quad x \in[-1,1]
$$

that has been studied in [33] with respect to equivariance solutions, consider the Cauchy problem with the initial condition

$$
y(0)=1 .
$$

We are interested here in the solutions $y \in C^{1}([-1,1],[-1,1])$ belonging to the class

$$
\mathscr{C}_{4}=\left\{y \in C([0,1],[0,1]):\left|y\left(t_{1}\right)-y\left(t_{2}\right)\right| \leq 4\left|t_{1}-t_{2}\right|, \forall t_{1}, t_{2} \in[0,1]\right\} .
$$

In this case, we have $L=4, a=-1, b=1, x_{0}=0$, and hence $C_{x_{0}}=\max \left\{x_{0}-\right.$ $\left.a, b-x_{0}\right\}=1$.

The function

$$
f(x, u)=\frac{1}{10} u^{2}
$$

is Lipschitzian with the Lipschitz constant $L_{1}=\frac{1}{5}$. Therefore, we have

$$
L_{1} C_{x_{0}}(L+1)=1 \text {, }
$$

so condition (3.5) is satisfied but condition (3.3) is not. Therefore, by Theorem 3.3, it follows that the initial value problem (4.2), (4.3) has at least one solution in $\mathscr{C}_{4}$ that can be approximated by means of the iterative method

$$
y_{n+1}(t)=(1-\mu) y_{n}(t)+\mu y_{0}+\frac{\mu}{10} \int_{x_{0}}^{t}\left(y_{n}\left(y_{n}(s)\right)\right)^{2} d s, \quad t \in[-1,1], n \geq 1,
$$

where $\mu \in(0,1)$ and $y_{1} \in \mathscr{C}_{4}$ is arbitrary.

Example 5. Consider the initial value problem defined by the iterative differential equation in Example 3 on the interval $[1 / 2,1]$ and the initial condition

$$
y\left(\frac{1}{2}\right)=\frac{1}{2} .
$$

We are interested here to study the solutions $y \in C^{1}([1 / 2,1],[1 / 2,1])$ belonging to the class $\mathscr{C}_{1,1}$. In this case, we have $L=1, a=1 / 2, b=1, x_{0}=1 / 2, y_{0}=1 / 2$ and $L_{1}=1$. 
In order to have (i) satisfied, we need $\lambda=1$ and hence, by (iii), $M=1$. Conditions (iv) and (v) are also satisfied, while the two conditions in (vi) reduce to $\bar{\tau}>0$ and, respectively, to $\frac{1-e^{-\frac{\bar{\tau}}{2}}}{\bar{\tau}} \leq \frac{1}{2}$, which always have solutions. This becomes obvious if we rewrite the second inequality equivalently as

$$
2 e^{-\frac{\bar{\tau}}{2}}+\bar{\tau} \geq 2
$$

Thus, all conditions in Theorem 3.4 are satisfied. A solution of the initial problem in $\mathscr{C}_{1,1}$ is $y(x)=\frac{1}{2}, x \in\left[\frac{1}{2}, 1\right]$. Note that the conditions in Theorem 3.4 are not satisfied on the whole interval [0,1], as $\mathscr{C}_{1,1}$ is a proper subclass of $\mathscr{C}_{1}$.

Remark 4. In a similar way, by exploiting the technique of non-expansive operators presented in this paper, we can study the iterative differential equations in [9-11, 17, 25, 29,33], or the functional differential equations with modified argument like those in [20,21], for which the technique of Picard operators, described in detail in [24, 26-28], is basically used. For example, in the very recent paper [17], a Picard type existence and uniqueness theorem it is obtained for iterative differential equations of the form

$$
y^{\prime}(x)=f(x, y(h(x)+g(y(x)))),
$$

a special case of which is the differential equation involved in the initial value problem (3.1) studied in the present paper. As such iterative differential equations are used to model infective disease processes, pattern formation in the plane, and are important in investigations of dynamical systems, future works will be also devoted to them.

\section{REFERENCES}

[1] C. Bacoţiu, "Volterra-Fredholm nonlinear systems with modified argument via weakly Picard operators theory," Carpathian J. Math., vol. 24, no. 2, pp. 1-9, 2008.

[2] M. Benchohra and M. A. Darwish, "On unique solvability of quadratic integral equations with linear modification of the argument," Miskolc Math. Notes, vol. 10, no. 1, pp. 3-10, 2009.

[3] V. Berinde, Iterative approximation of fixed points, 2nd ed., ser. Lecture Notes in Mathematics. Berlin: Springer, 2007, vol. 1912.

[4] A. Buică, "Existence and continuous dependence of solutions of some functional-differential equations," Seminar on Fixed Point Theory 3, pp. 1-14, 1995, a publication of the Seminar on Fixed Point Theory Cluj-Napoca.

[5] C. Chidume, Geometric properties of Banach spaces and nonlinear iterations, ser. Lecture Notes in Mathematics. London: Springer-Verlag London Ltd., 2009, vol. 1965.

[6] M. Dobriţoiu, "System of integral equations with modified argument," Carpathian J. Math., vol. 24, no. 2, pp. 26-36, 2008.

[7] M. Edelstein, "A remark on a theorem of M. A. Krasnoselski," Amer. Math. Monthly, vol. 73, pp. 509-510, 1966.

[8] E. Eder, "The functional-differential equation $x^{\prime}(t)=x(x(t))$," J. Differential Equations, vol. 54, no. 3, pp. 390-400, 1984. [Online]. Available: http://dx.doi.org/10.1016/0022-0396(84)90150-5

[9] E. Egri, "Cauchy problem for a system of iterative functional-differential equations," Analele Univ. Timişoara, Ser. Mat. Inf., vol. 46, no. 1, pp. 23-49, 2008. 
[10] E. Egri, "A boundary value problem for a system of iterative functional-differential equations," Carpathian J. Math., vol. 24, no. 1, pp. 23-36, 2008.

[11] E. Egri and I. A. Rus, "First order iterative functional-differential equation with parameter," Stud. Univ. Babeş-Bolyai Math., vol. 52, no. 4, pp. 67-80, 2007.

[12] M. Fečkan, "On a certain type of functional-differential equations," Math. Slovaca, vol. 43, no. 1, pp. 39-43, 1993.

[13] K. Goebel and W. A. Kirk, Topics in metric fixed point theory, ser. Cambridge Studies in Advanced Mathematics. Cambridge: Cambridge University Press, 1990, vol. 28. [Online]. Available: http://dx.doi.org/10.1017/CBO9780511526152

[14] S. Ishikawa, "Fixed points and iteration of a nonexpansive mapping in a Banach space," Proc. Amer. Math. Soc., vol. 59, no. 1, pp. 65-71, 1976.

[15] M. A. Krasnoselskii, "Two remarks on the method of successive approximations," Uspehi Mat. $\operatorname{Nauk}$ (N.S.), vol. 10, no. 1(63), pp. 123-127, 1955.

[16] W.-T. Li and S. Zhang, "Classifications and existence of positive solutions of higher order nonlinear iterative functional differential equations," J. Comput. Appl. Math., vol. 139, no. 2, pp. 351-367, 2002. [Online]. Available: http://dx.doi.org/10.1016/S0377-0427(01)00414-9

[17] W.-r. Li and S. S. Cheng, "A Picard theorem for iterative differential equations," Demonstratio Math., vol. 42, no. 2, pp. 371-380, 2009.

[18] A. Mureşan, "Step method for a functional-differential equation from mathematical economics," Carpathian J. Math., vol. 24, no. 3, pp. 356-362, 2008.

[19] V. Mureşan, "Step method for a functional-differential equation in Banach space," Carpathian J. Math., vol. 24, no. 3, pp. 363-369, 2008.

[20] V. Mureşan, Ecuaţii diferenţiale cu modificarea afină a argumentului [Differential equations with affine modification of the argument]. Cluj-Napoca: Transilvania Press, 1997, in Romanian.

[21] V. Mureşan, Functional-integral equations. Editura Mediamira, Cluj-Napoca, 2003.

[22] A. Rontó and M. Rontó, "On a Cauchy-Nicoletti type three-point boundary value problem for linear differential equations with argument deviations," Miskolc Math. Notes, vol. 10, no. 2, pp. 173-205, 2009

[23] A. Rontó and M. Rontó, "Successive approximation method for some linear boundary value problems for differential equations with a special type of argument deviation," Miskolc Math. Notes, vol. 10, no. 1, pp. 69-95, 2009.

[24] I. A. Rus, "A fiber generalized contraction theorem and applications," Mathematica, vol. 41(64), no. 1 , pp. 85-90, 1999.

[25] I. A. Rus, "A class of nonlinear functional-integral equations via weakly Picard operators," An. Univ. Craiova Ser. Mat. Inform., vol. 28, pp. 10-15, 2001.

[26] I. A. Rus, Generalized contractions and applications. Cluj University Press, Cluj-Napoca, 2001.

[27] I. A. Rus, "Weakly Picard operators and applications," Semin. Fixed Point Theory Cluj-Napoca, vol. 2, pp. 41-57, 2001.

[28] I. A. Rus, "Picard operators and applications," Sci. Math. Jpn., vol. 58, no. 1, pp. 191-219, 2003.

[29] I. A. Rus and E. Egri, "Boundary value problems for iterative functional-differential equations," Stud. Univ. Babeş-Bolyai Math., vol. 51, no. 2, pp. 109-126, 2006.

[30] H. Schaefer, "Über die Methode sukzessiver Approximationen," Jber. Deutsch. Math. Verein., vol. 59, no. Abt. 1, pp. 131-140, 1957.

[31] B. H. Stephan, "On the existence of periodic solutions of $z^{\prime}(t)=-a z(t-r+\mu k(t, z(t)))+$ $F(t)$," J. Differential Equations, vol. 6, pp. 408-419, 1969.

[32] K. Wang, "On the equation $x^{\prime}(t)=f(x(x(t)))$," Funkcial. Ekvac., vol. 33, no. 3, pp. 405-425, 1990. [Online]. Available: http://www.math.kobe-u.ac.jp/ fe/xml/mr1086769.xml 
[33] D. Yang and W. Zhang, "Solutions of equivariance for iterative differential equations," Appl. Math. Lett., vol. 17, no. 7, pp. 759-765, 2004. [Online]. Available: http: //dx.doi.org/10.1016/j.aml.2004.06.002

Author's address

Vasile Berinde

North University of Baia Mare, Department of Mathematics and Computer Science, 76 Victoriei St., 430122 Baia Mare, Romania

E-mail address: vberinde@ubm.ro 